\title{
PERFORMANCE EVALUATION OF BROADCASTING PROTOCOLS FOR AD HOC AND SENSOR NETWORKS
}

\author{
Hong Guo ${ }^{1}$, François Ingelrest ${ }^{2}$, David Simplot-Ryl ${ }^{2}$, \\ and Ivan Stojmenović ${ }^{1}$ \\ ${ }^{1}$ Computer Science, SITE. University of Ottawa, Canada. \\ hguo023yahoo.ca, ivan@site.uottawa.ca \\ ${ }^{2}$ IRCICA/LIFL, University of Lille 1, France. INRIA futurs, POPS research groups. \\ Francois.Ingelrest@lifl.fr, David.simplot@lifl.fr
}

\begin{abstract}
Many broadcasting protocols for ad hoc and sensor networks have been proposed. Multipoint relay (MPR) and dominating set (DS) schemes can effectively improve the efficiency while providing reliable broadcasting. The neighbor elimination scheme (NES) can improve any broadcasting protocol as an added feature. We evaluate the performance of MPR (source dependent), MPRDS (source-independent MPR), and DS-based protocols. We add NES to these schemes separately and evaluate the performance of the resulted protocols. As a result, DS-NES appears to be the most robust, taking all measurements and parameters into acount, because it remains competitive under all scenarios, and has significant advantages over MPR-DS-NES in dynamic scenarios, and over MPR-NES when the broadcast message is not very large, because MPR has overhead in packet lengths.
\end{abstract}

\section{Introduction}

Ad hoc and sensor networks are two kinds of wireless networks operating without infrastructure, relying on hosts for communication tasks. Among the common problems found in these two kinds of networks is broadcasting, which can be used for route discovery for example. It is a well-known one-to-all communication task, where one host $u$ whishes to send a given set of data to all the other ones. Since normally the source node is not within transmission radius to all the recipient nodes, many hosts will have to act as routers for the task to be achieved. The easiest way is to have all nodes act as routers and retransmit the messages at least once to their neighborhood: this is a protocol known as blind flooding. In networks which are not sparse, it generates a lot of collisions 
that could possibly prevent the broadcasting from being correctly performed. Moreover, significant energy is consumed by the redundant messages. A number of other schemes have been proposed to replace blind flooding, and they can be classified in different categories: simple flooding, probability based, area based and neighbor knowledge methods.

In this paper, we aim at evaluating the performances of protocols from the fourth category only. Indeed, for the existing probability and area based protocols, the performances of the protocols are closely related to the predetermined parameters and thresholds for which the best values may depend on network conditions. Moreover, they are not reliable as illustrated by [Stojmenović et al., 2001]. The reliability of a broadcasting protocol refers to the capability of reaching all the nodes in the network when considering a collision free environment. Neighbor knowledge methods normally provide reliable broadcasting, and can be further divided into self-pruning and neighbor-designating methods, according to whether a node makes a local decision to retransmit a broadcast packet or is told by the upstream sender (either via the packet or via a previously sent control packet) whether it needs to retransmit the packet. We may also refer to these two types of methods as source-dependent and sourceindependent methods. From these two behaviors, we chose the multipoint relay protocol (MPR) proposed by [Qayyum et al., 2002] and the generalized selfpruning rule presneted by [Dai and $\mathrm{Wu}, 2003$ ] as they are both efficient and representative. A variant combining MPR and dominating sets, namely MPRDS proposed by [Adjih et al., 2005], is also studied. Secondly, by adding the neighbor elimination scheme studied by [Peng and Lu, 2000] and by [Stojmenović et al., 2001] to the above mentioned schemes, we are able to illustrate that it improves the performance of any broadcasting protocol as added feature.

The remainder of this paper is organized as follows: in the next section, we provide the definitions needed by our network model, while in Sec. 3 are provided the technique, algorithms and procedures used in our simulations, as well as the assumptions made for our experiments and the obtained results. We finally conclude in Sec. 4 and provide some directions for future research.

\section{Preliminaries}

We represent an ad hoc network by a graph $G=(V, E)$ where $V$ is the set of nodes and $E \subseteq V^{2}$ is the set of edges that gives the available communications: $(u, v)$ belongs to $E$ means that $v$ is a physical neighbor of $u$, i.e. $u$ can directly send a message to $v$. Let us assume that the maximum range of communication, denoted by $R$, is the same for all vertices and that $d(u, v)$ is the Euclidean distance between $u$ and $v$. The set $E$ is then defined as follows:

$$
E=\left\{(u, v) \in V^{2} \mid d(u, v) \leq R\right\} .
$$


So defined graph is called the unit graph, with $R$ as its transmission radius. Each node $u \in V$ is assigned a unique value to be used as an identifier (id), so that the identifier of $u$ is denoted by id $(u)$. We also define the neighborhood set $N(u)$ of a vertex $u$ as:

$$
N(u)=\{v \mid(u, v) \in E\} .
$$

The size of this set, $|N(u)|$, is also known as the degree of $u$. The density of the graph is the average degree for each node. Note that $(u, u)$ is not in $E$.

The distance between two nodes is measured in term of number of hops, which is simply the minimum number of links to cross from a source node to a destination one.

Nodes in a broadcasting protocol may require various neighborhood information. The protocols considered in this article require 2-hop topological information at each node. It may be obtained by two rounds of 'HELLO' messages, to send information about itself to neighbors, and to send collected information about its neighbors so that each node can acquire 2-hop knowledge. One of the selected protocols, MPR-DS, requires the third round of 'HELLO' messages, so that each node can inform all its neighbors about forwarding decisions, which are used later when a broadcasting task emerges.

Extended literature review and more explanations on our simulations design can be found in [Guo et al., 2005].

\section{Performances Evaluation}

In our research, the experiments were carried out in two phases: in the first one, the performance of the basic MPR, CDS and MPR-DS schemes were evaluated. In the second one, we added a NES to these algorithms and evaluated the performance of the resulting methods. We used random uniform unit disc graphs to model ad hoc and sensor networks, and considered only connected topologies. We adopt certain assumptions to appropriately define the area of our study. These assumptions can be summarized as follow:

- An ideal MAC protocol (no collision) is used, and nodes are static. Thus, any effect that mobility may have is avoided: because of localized algorithms being applied, it is assumed that relative positions of nodes do not change (sufficiently to impact the performance) while broadcasting.

- There is only one broadcasting task at a time in the network and no other message traffic exists. We thus avoid the impact of collisions, believing that a protocol with a lower overhead on one broadcasting task reduces collision impact on other tasks and is thus expected to perform better if collision were added. 
- There is synchronization among the transmissions, channel is time-slotted and each transmission takes one slot.

- Each time a node transmits a packet, all its 1-hop neighbors receive it.

- While a node transmits, none of its neighbors up to 2-hop are transmitting. This assumption was used to eliminate the problem of interference when a node receives two radio transmissions at the same time by two of its neighbors, which are not neighbors themselves.

We used the rule by Dai and Wu to compute CDS's. We define the priority of a node with a record key $=($ degree, id $)$ : nodes compare their degrees first and the node with the higher degree has greater chances of being in the connected dominating set. In case of ties, the node with the highest id has priority to be selected. We used the same priority for MPR-DS and the enhancement by Wu.

We call a node that is in multipoint relay set or in connected dominating set a relay candidate. There are two factors that a relay candidate $c$ needs to consider before it relays the broadcast packet when using NES: timeout and forwardingList. Upon the first reception of the broadcast packet, $c$ sets up timeout $=(1 /$ numberCoverd, id $)$, where numberCovered is the number of 1 hop neighbors who have not received the packet after the same transmission. In case of ties, the node with the lowest id will rebroadcast the packet first. The forwardingList, at first, contains all 1-hop neighbors of $c$. For each reception of the packet, $c$ eliminates from the forwardingList all neighbors receiving the packet from the same transmission. If $c$ gets a packet from one of its neighbors after its first reception, it may need to adjust its original timeout when the number of uncovered neighbors changes. When timeout expires, $c$ will rebroadcast the packet if its forwardingList is not empty. When adding a neighbor elimination scheme within a multipoint relaying broadcasting protocol, instead of letting all existing MPR's compute their own MPR's, we decided that only the MPR's which relay the packet to their neighbors carry out further computation. That means, if a MPR $v$ has an empty forwarding list, $v$ will not rebroadcast the packet and thus will not compute its own MPR's.

We first measure the percentage of re-transmitting nodes (PRN). To do this, the number of nodes that rebroadcasts the message is counted, and compared to the total number of nodes. Fig. 1 illustrates the simulation results. Subfigure 1(a) indicates that MPR has a lower ratio compared to DS and MPR-DS. However, each message in MPR is of longer size and therefore the selection of method with lowest overall packet size depends on the size of broadcast packet with respect to the size of neighbor's id. DS and MPR-DS behave equally well. This observation is consistent with results by [Adjih et al., 2005].We observe that the neighbor elimination scheme has improved DS and MPR-DS significantly, but does not seem to have a significant impact on MPR when the 


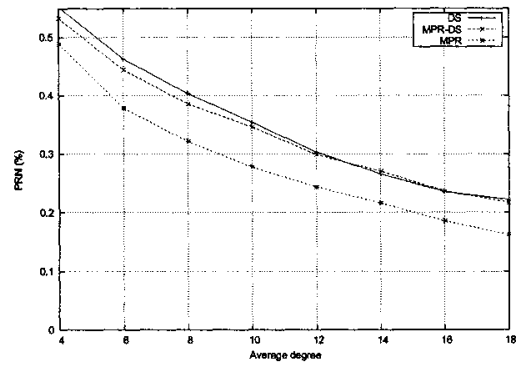

(a) All schemes.

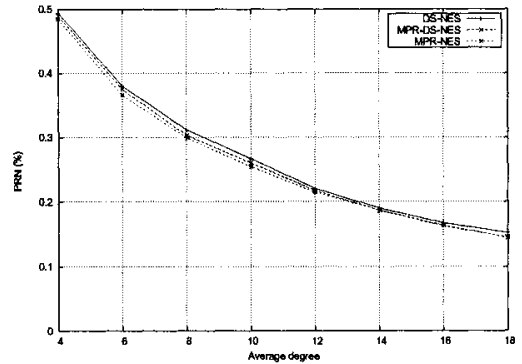

(b) All schemes with NES.

Figure 1. PRN versus average degree.

average node degree is less than 10 and only does trivial improvement to MPR for $d>10$. It is interesting to note that after adding the neighbor elimination scheme, three new protocols behave almost equally well.

Our result reveals that all algorithms depend on the density of the network. In sparse networks, more nodes need to rebroadcast in order to reach all the nodes in the network. As the density increases, proportionally fewer nodes rebroadcast. This observation differs from the result by [Stojmenović et al., 2001], where it has been observed that the ratios appear to be relatively stable with respect to degree $d$. We argue that our result is more reasonable because when the degree $d$ increases, the number of neighbors covered by one transmission increases, consequently the number of retransmissions needed to cover a certain number of nodes ( $n=100$ in our case) decreases.

We also measure the number of nodes that each transmission covers. In fact this number is the number of 1-hop neighbors of each transmitting node. An average value on all the transmitting nodes is computed and compared with other methods. In Fig. 2(a) and 2(b), it is observed that NFN increases with respect to the average node degree $d$ for all the methods. This was predictable as theoretically the NFN is closely related to $d$. DS has shown superiority over MPR and MPR-DS while DS-NES performs a little better than MPR-NES and MPR-DS-NES. Recall that in MPR, a node is chosen to be a multipoint relay because it covers a maximal number of un-covered neighbors. Although this number relies on the node degree to a certain extent, a node which covers the most uncovered neighbors will not necessarily have the highest degree in its neighborhood. However in DS, nodes with higher degrees have a higher priority to be in the connected dominating set. This is also true for MPR-DS most of the time. Notwithstanding the previous statement, in MPR-DS, a node with the highest degree in its neighborhood but without being an intermediate 


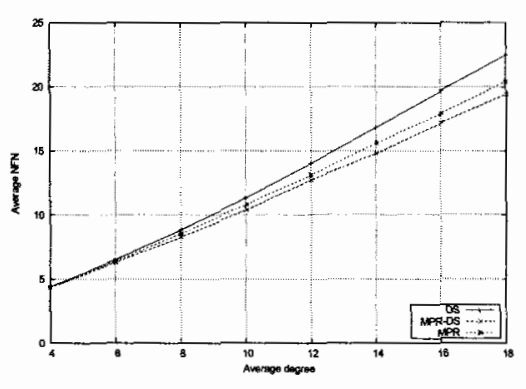

(a) All schemes.

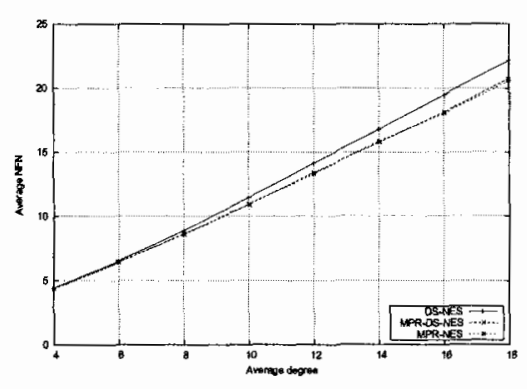

(b) All schemes with NES.

Figure 2. NFN versus average degree.

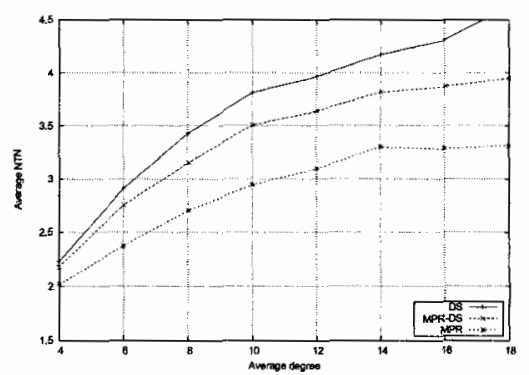

(a) All schemes.

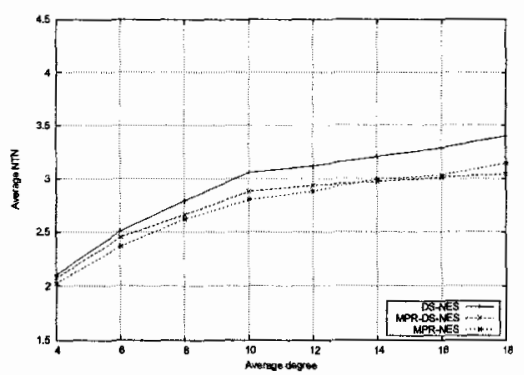

(b) All schemes with NES.

Figure 3. NTN versus average degree.

node cannot be in the connected dominating set. Ergo, there is the possibility that node with highest degree is not selected for both MPR and MPR-DS. Consequently, DS has a larger coverage per transmission.

The neighbor elimination scheme does not have significant impact on the average number of nodes covered by each transmitting nodes. This demonstrates that the neighbor elimination scheme improves the broadcasting protocols by reducing redundant retransmissions.

We now give results concerning the number of times each non-transmitting node receives the message, noted NTN. From Fig. 3, we observe that, in sparse networks, non-transmitting nodes get less redundant messages. While the degree $d$ increases, each node receives more copies of the same message. The neighbor elimination scheme effectively reduces the redundancy in the network. 


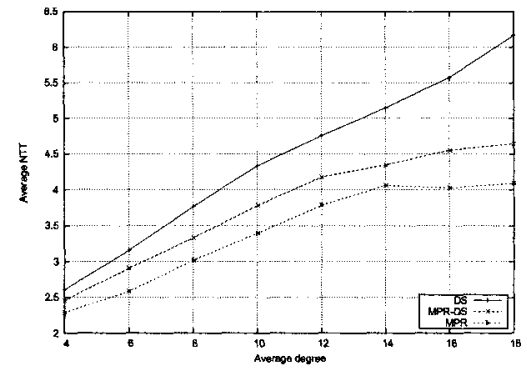

(a) All schemes.

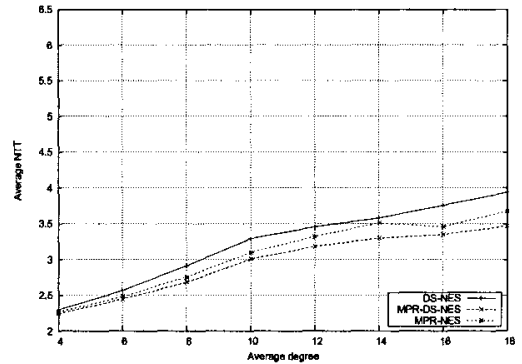

(b) All schemes with NES.

Figure 4. NTT versus average degree.

Fig. 3 presents the measured NTN for all methods under consideration. We see that MPR has a better performance than DS and MPR-DS (each node receiving fewer copies of the same packet), while MPR-DS lies in-between DS and MPR. From 3(b), we observe that after adding the neighbor elimination scheme, the three methods tend to have similar performances. The neighbor elimination scheme reduces NTN, thus reducing the traffic in the network. This improvement is more obvious for DS and MPR-DS protocols.In these two methods, on average, NTN has been reduced by 0.5 to 1 in dense networks (when $d=8$ ). But, MPR-NES still has slightly better performance than DS-NES and MPR-DS-NES overall.

We now give results about the number of times each transmitting node receives the message, noted NTT. An observation similar to NTN can be obtained for NTT from Fig. 4. That is, there is less redundancy in sparse networks than dense networks. Recall that with the average degree $d$ increases, fewer nodes retransmit the message. However, the number of times each node, transmitting or non-transmitting, receives the same message increases. This can be explained by the increased coverage of each transmission. According to our observations, it appears that the transmitting nodes receive more copies of the same message than non-transmitting nodes. Once again, we notice that the neighbor elimination scheme improves DS and MPR-DS more than on MPR. In fig. 4, MPR exhibits the best performance on NTT. DS has the most redundancy on transmitting nodes. With the help of neighbor elimination scheme, MPR-DS-NES outperforms MPR-NES and DS-NES for most $d$ values, as indicated in 4(b).

We finally consider the overhead brought by MPR scheme in the size of broadcast messages by including ids of relays in these messages. The other 


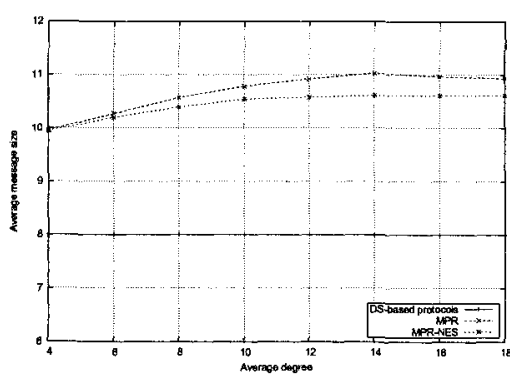

(a) $P=8$.

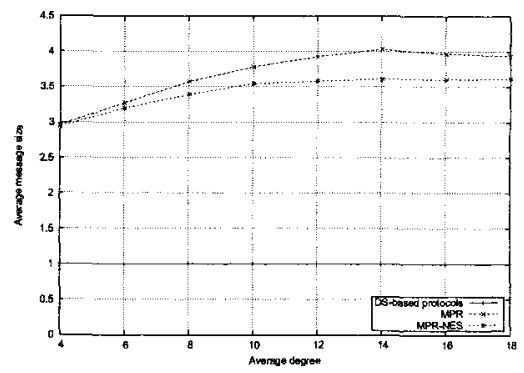

(c) $P=1$.

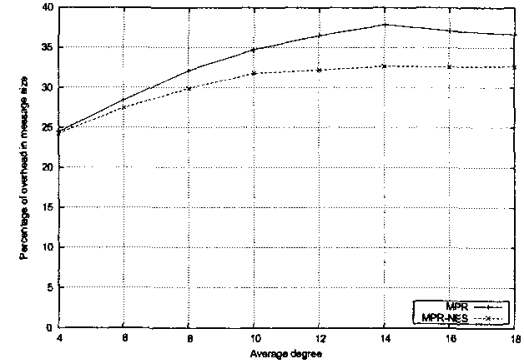

(b) $P=8$.

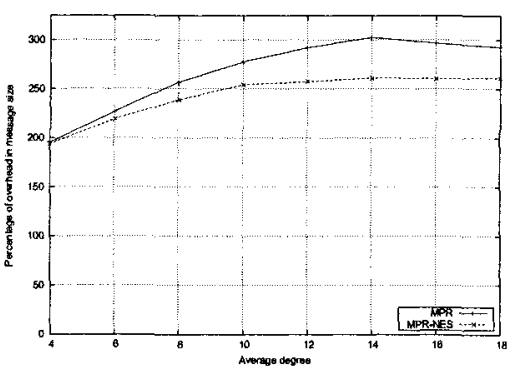

(d) $P=1$.

Figure 5. Average message size and overhead in message size for MPR scheme.

schemes, based on CDS, do not need to forward additional information within the broadcast packet. Let the unit packet size be equal to the size of the id of one neighbor in the forwarding list. Let $p$ be the size of broadcast message in such units. Each MPR message is of different length, which is $p+s, s$ being the number of neighbors in forwarding list, while dominating set approaches need $p$ size for each message. We give in Fig. 5 the comparison between the different schemes for $p=1$ and $p=8$. We first measured the average size of each message being transmitted, and the percentage of overhead in the size of the transmitted message. We can observe that the overhead brought by the inclusion of ids of relays can be rather huge if the original size is small. For $p=8$ in 5(a) and 5(b), the overhead ranges from $25 \%$ to $40 \%$ for densities between 4 and 18, while for $p=1$ in 5(c) and 5(d), it ranges between $200 \%$ and $300 \%$, compared to dominating set based approaches.

To complete the study of message overhead, we then measured the message dilation, as the ratio of overall message sizes transmitted by given protocol 


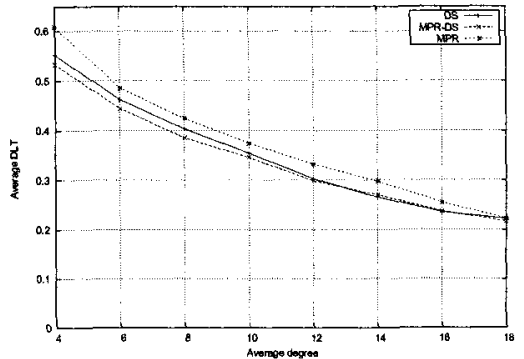

(a) $P=8$ without NES.

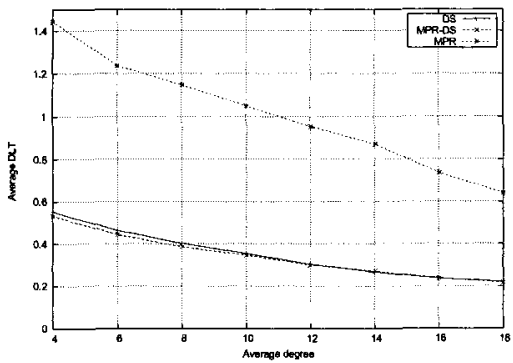

(c) $P=1$ without NES.

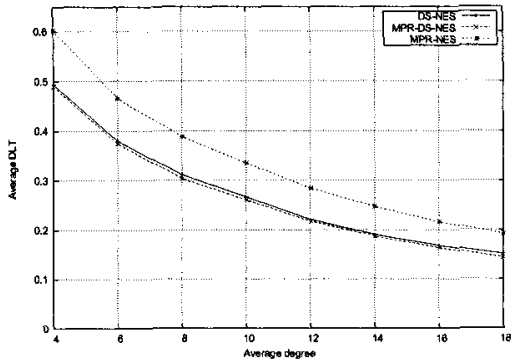

(b) $P=8$ with NES.

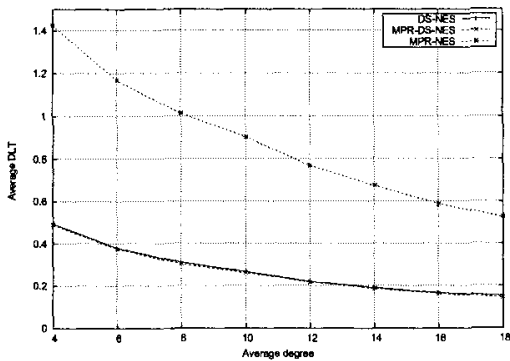

(d) $P=1$ with NES.

Figure 6. Average dilation for the miscellaneous schemes.

with respect to the overall message size used in blind flooding solution. The latter is $n p$, where $n$ is the number of nodes in the network. We thus give in Fig. 6 the value of this ratio for the values $p=1$ and $p=8$. It confirms that for small value of $p$, the overhead brought by the inclusion of MPR relays is rather huge, while the two schemes based on CDS are very near from each other.

We can infer that CDS-based schemes are superior to MPR scheme when $p$ has a small value, because of the significant overhead (and consequently the number of collisions at the MAC layer) in MPR, while the difference will become negligible for higher values of $p$ (for example when broadcasting large files).

An interesting observation is that the notable improvement on MPR, by adding the neighbor elimination scheme, starts at $d=10$. This happens because in sparse networks more relaying nodes need to rebroadcast to reach isolated neighbors. 


\section{Conclusion}

We have seen that the NES can enhance the performances of all the protocols as an added feature, especially in dense networks. The notable improvement on MPR starts at $d=10$ : in sparse networks, more relaying nodes need to relay to reach isolated nodes. Examples are the neighbors of a node with degree one, and such nodes are more likely to exist in sparse networks than in dense ones. NES improves DS and MPR-DS protocols more significantly than MPR, revealing that source-independent protocols benefit more from NES than source-dependent ones.

Among all the schemes, MPR-NES appears to require the least number of retransmissions, but the advantage is not major. However, this is balanced by the increased size of messages, which can cause more collisions and requires more energy. For smaller packet sizes, CDS-based protocols appear superior: MPR-DS-NES protocol performs slightly better than DS-NES. However, it requires a third round of HELLO messages, and is therefore inferior in dynamic networks. It appears that pure DS-based methods approaches are overall winning ones, remaining competitive under all scenarios and having huge advantage in dynamic networks or with smaller packets.

All algorithms depend on the density of the network: in sparse ones, more nodes need to relay to reach all the nodes but as the density increases, proportionally fewer nodes need to rebroadcast while the number of times each node, transmitting or not, receives the same message increases. Transmitting nodes receive more copies of the same message than non-transmitting ones for all $d$ values.

\section{References}

[Adjih et al., 2005] Adjih, C., Jacquet, P., and Viennot, L. (2005). Computing connected dominated sets with multipoint relays. Ad Hoc \& Sensor Wireless Networks, 1(1 - 2):27 - 39.

[Dai and Wu, 2003] Dai, F. and Wu, J. (2003). Distributed dominant pruning in ad hoc networks. In Proceedings of the IEEE International Conference on Communications (ICC'03), Anchorage, Alaska.

[Guo et al., 2005] Guo, H., Ingelrest, F., Simplot-Ryl, D., and Stojmenović, I. (2005). Performance evaluation of broadcasting protocols for ad hoc and sensor networks. Technical report, INRIA.

[Peng and Lu, 2000] Peng, W. and Lu, X. (2000). On the reduction of broadcast redundancy in mobile ad hoc networks. In Proceedings of the ACM MobiHoc 2000, Boston, USA.

[Qayyum et al., 2002] Qayyum, A., Viennot, L., and Laouiti, A. (2002). Multipoint relaying for flooding broadcast messages in mobile wircless networks. In Proceedings of the Hawaii International Conference on System Sciences (HICSS'02), Big Island, Hawaii.

[Stojmenović et al., 2001] Stojmenović, I., Seddigh, M., and Zunic, J. (2001). Dominating sets and neighbor elimination-based broadcasting algorithms in wireless networks. IEEE Transactions on Parallel and Distributed Systems, 13(1):14- 25. 